\title{
Convective Heat Transfer in a Square Cavity with a Heat-Generating Body of Different Aspect Ratios
}

\author{
Samuel I. Afolabi*, Anthony O. Ojo, Modupe A. Oluleye, Ayotunde A. Ojo \\ Department of Mechanical Engineering \\ Ekiti State University, Ado-Ekiti, PMB 5363, Nigeria
}

\begin{abstract}
The problem of flow and heat transfer in a square cavity with a heat source at the center has been numerically studied. For the cavity considered, the horizontal walls are insulated while the vertical walls are maintained at constant cold temperature and the heat source at the center is kept at a constant hot temperature. The principal partial differential equations of mass, momentum, and energy are solved by applying Finite Element Method to perform a parametric study. Numerical experiments was conducted for Rayleigh numbers $(R a)$ in the range; $10^{2}$ to $10^{7}$ for a heat generating (source) body of aspect ratios $(A R)=0.1-0.6$ with steps of 0.1. Streamlines and Isotherm contours of the ensuing temperature and flow fields are presented together with the estimated Nusselt numbers $\mathrm{CNu}$ ) for a fluid (air at room temperature) with Prandtl number of 0.71. The results of the numerical experiment indicate that for a fixed AR, with increasing $R a$, the heated fluid is transported to the top-half of the cavity; a region characterized by increased fluid motion due to convection of the less dense heated fluid. From the estimated $\mathrm{Nu}$ along the boundary of the heat source in this top-half region, heat transfer by convection is a generally dominant heat transfer mechanism, however, with increasing $A R$ and due to decreasing mid-cavity temperature gradients, contributions of heat conduction to the fluid is steadily noticeable as a wide section of the obstacle is in contact with the fluid.
\end{abstract}

Keywords - Natural convection; Square cavity; Galerkin weighted residual; Heat transfer; conducting body

\section{INTRODUCTION}

Convective flow and heat transfer in a square cavity have been extensively investigated for decades [1]. Results from such studies have contributed greatly to the development and optimization of a range of engineering applications and computational methods. In practice, the subject of flow and heat transfer in a square cavity finds importance in the electronic systems, where the phenomenon of natural convection is utilized for the purpose of cooling an assemblage of components in an enclosed system. Also, natural convection finds wide usage in the nuclear reactors, heating, ventilation, air-conditioning (HVAC) systems, and in various applications like solar collectors, cooling of mechanical parts and in thermal storage facilities [2, 3, 4]. In these applications, the main heat transfer mechanism is natural convection, so, it is imperative to know how the transport of flow and heat affect the operation of such applications [5].

As earlier mentioned, convective heat transfer in square cavities have been widely investigated, with these studies providing insight on the transport of both flow and heat within these cavities. These studies are largely computational in nature where a variety of numerical strategies have been implemented for various geometry and boundary conditions. Natural convection in a square cavity was investigated using a high-resolution finite difference method (FDM) [6], with the study showing how increasing internal heat generation affects the thermal energy in the cavity. Using a differential quadrature method, [7] examined convection in cavities having one of the vertical walls heated and the other cooled with both horizontal walls insulated. Results from the study show that with variation of the aspect ratios of the cavity, there is an increase in vorticity with increasing Rayleigh number. Reference [8] examined the effect of uniform and non-uniform wall temperatures on convection in square enclosure using Galerkin Finite Element Method (FEM). Their study that heat transfer was enhanced due to the uniform temperature boundaries than that of the non-uniform temperature case. Reference [9] investigated the flow and heat transfer in a square enclosure that has a heat generating conducting body within it together with a temperature difference across the cavity. In that study, the ratio of the average Nusselt number of the vertical walls drastically changes due to convection parameters occasioned by the difference in temperature through the enclosure. Reference [10] studied the convective flow and heat transfer phenomena in a cavity with differentially heated side walls at different Rayleigh numbers $(R a)$. Results from that study demonstrated that the stream function-vorticity finite difference and lattice Boltzmann formulations are effective in providing insight to the convective phenomena in the enclosure. The effect of magnetic field in a square cavity with semi-circular heated body has been investigated [11]. The study showed that with increase in Rayleigh number, to decrease free convection in the cavity, a stronger magnetic field is needed compared to the cases where lower Rayleigh numbers are considered. Reference [1] studied natural convection in a square cavity with a non-uniformly heated bottom wall and a square shape heated body and it was shown that both temperature and flow distribution in the cavity hinge on both the strength of the magnetic field and Rayleigh number.

This study aims at investigating the flow and heat transfer phenomenon in a case of two temperature differentials caused by that of the square cavity enclosure and that of a heatgenerating conducting obstacle of different aspect ratios. For this study, the horizontal walls are insulated while the vertical walls are maintained at constant cold temperature and the heat source at the center is kept at a constant hot temperature. By 
considering various Rayleigh numbers and aspect ratios, numerical simulation is performed with a FEM that uses the Galerkin weighted residuals approach.

\section{METHODS AND MATERIALS}

Fig. 1 shows a schematic of the cavity having a heat generating conducting body located at its center. The aspect ratio $(\mathrm{AR}=l / \delta)$ of this heat source is in the range; 0.1 to 0.6 with steps of 0.1 . The horizontal walls of the cavity are insulated while the vertical walls are maintained at constant cold temperature and the heat source at the center is kept at a constant hot temperature. It is assumed that the enclosure is filled with air with $\operatorname{Pr} \sim 0.71$.

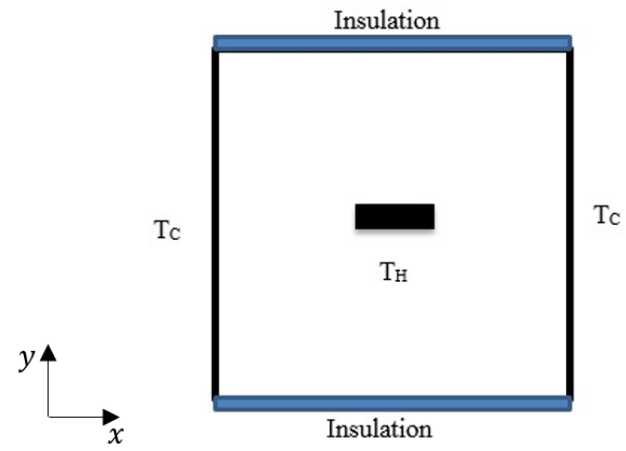

Fig. 1. Square cavity with a heat source at the center.

This study considered a Newtonian fluid with constant properties except density and also a steady state incompressible flow. The fundamental governing equations for the conservation of mass, momentum, and energy can be written as:

$$
\frac{\partial u}{\partial x}+\frac{\partial v}{\partial y}=0
$$

$u \frac{\partial u}{\partial x}+v \frac{\partial u}{\partial y}=-\frac{1}{\rho} \frac{\partial P}{\partial x}+\vartheta\left(\frac{\partial^{2} u}{\partial x^{2}}+\frac{\partial^{2} u}{\partial y^{2}}\right)$

$u \frac{\partial v}{\partial x}+v \frac{\partial v}{\partial y}=-\frac{1}{\rho} \frac{\partial P}{\partial y}+\vartheta\left(\frac{\partial^{2} v}{\partial x^{2}}+\frac{\partial^{2} v}{\partial y^{2}}\right)+\beta g\left(T_{h}-T_{c}\right)$

$u \frac{\partial T}{\partial x}+v \frac{\partial T}{\partial y}=\propto\left(\frac{\partial^{2} T}{\partial x^{2}}+\frac{\partial^{2} T}{\partial y^{2}}\right)$

In order to model the heat and flow transport in the square cavity, a computer simulation environment of COMSOL Multiphysics was used. It's a flexible platform that allows modeling of relevant physical aspects. The parameterized geometry and custom solver sequences were adopted to solve dimensionless nonlinear governing partial differential equations of the conservations of mass, momentum, and energy equations using the Galerkin weighted residual FEM. The adapted nonlinear equations are transformed into linear algebraic equations with the aid of Newton's iteration strategy in COMSOL Multiphysics software while the fully coupled system is set to perform 50 iterations. With 8206 mesh elements and 304 boundary elements, a total of 29485 degree of freedom were solved in addition to 612 internal degree of freedom. It then repeatedly solved problems of buoyant flow in the square cavity and analyzed different temperature profiles as well as the convective flow patterns. The factor in error estimate is 400 while the convergence tolerance is $10^{-6}$. The iterative procedure is adjusted for an effective solution using the dimensionless parameters defined below and a Buosinessq approximation for the buoyant force.

The following dimensionless quantities are used to nondimensionlize equations (1)-(4) as follows;

$$
\begin{aligned}
& \mathrm{X}=\frac{x}{\delta}, \Omega=\frac{\omega \delta^{2}}{\alpha}, \mathrm{Y}=\frac{y}{\delta}, U=\frac{\mathrm{u} \delta}{\alpha}, V=\frac{\mathrm{v} \delta}{\alpha}, \\
& \theta=\frac{T-T_{c}}{T_{H}-T_{r}}, \quad \mathrm{Pr}=\frac{\vartheta}{\alpha}, R a=\frac{\beta g\left(T_{H}-T_{c}\right) \delta^{3} P r}{\vartheta^{2}}, N u=\frac{h l}{k}
\end{aligned}
$$

The dimensionless equations are;

$$
\begin{aligned}
& U \frac{\partial \Omega}{\partial X}+V \frac{\partial \Omega}{\partial Y}=\operatorname{Pr}\left(\frac{\partial^{2} \Omega}{\partial X^{2}}+\frac{\partial^{2} \Omega}{\partial Y^{2}}\right)+\operatorname{Ra} \cdot \operatorname{Pr} \frac{\partial \theta}{\partial X} \\
& U \frac{\partial \theta}{\partial X}+V \frac{\partial \theta}{\partial Y}=\frac{\partial^{2} \theta}{\partial X^{2}}+\frac{\partial^{2} \theta}{\partial Y^{2}}
\end{aligned}
$$

\section{Boundary Conditions}

It is assumed that the no-slip condition applies for the walls of the cavity and for the walls of heat-generating conducting body. In addition to this, it is assumed that there is thermal equilibrium between the fluid and all the walls.

Velocity;

$$
\begin{array}{r}
\boldsymbol{u}(\boldsymbol{x}, 0)=\boldsymbol{u}(\boldsymbol{x}, L)=\boldsymbol{u}(\mathbf{0}, y)=\boldsymbol{u}(L, y)=\mathbf{0} \\
\boldsymbol{v}(\boldsymbol{x}, 0)=\boldsymbol{v}(\boldsymbol{x}, L)=\boldsymbol{v}(\mathbf{0}, y)=\boldsymbol{v}(L, y)=\mathbf{0}
\end{array}
$$

Temperature;

Left wall, $\theta(0, y)=T_{c} \quad$ Right wall, $\theta(L, y)=T_{c}$

Top wall, $\boldsymbol{\theta}(x, \boldsymbol{L})=\infty \quad$ Bottom wall, $\boldsymbol{\theta}(x, \mathbf{0})=\infty$

The mid-cavity obstacle of various $\mathrm{AR}, \boldsymbol{\theta}=\boldsymbol{T}_{\boldsymbol{H}}$

\section{PROGRAM VALIDATION AND COMPARISON WITH PREVIOUS WORK}

By using a geometry similar to that of [12], our program code was validated with the numerical experiments of [12]. This geometry was that of a square cavity having an adiabatic obstacle of $\mathrm{AR}=0.4$ located at the middle of the cavity. The left vertical wall is maintained at high temperature, the right wall is kept at cold temperature, while the upper wall and 
bottom wall is adiabatic. The streamlines and temperature profiles obtained with our numerical setup for $R a=10^{3}$ and $10^{4}$ are plotted in Figs. 2 and 3 and this shows an excellent agreement with the case study of [12].

(a)
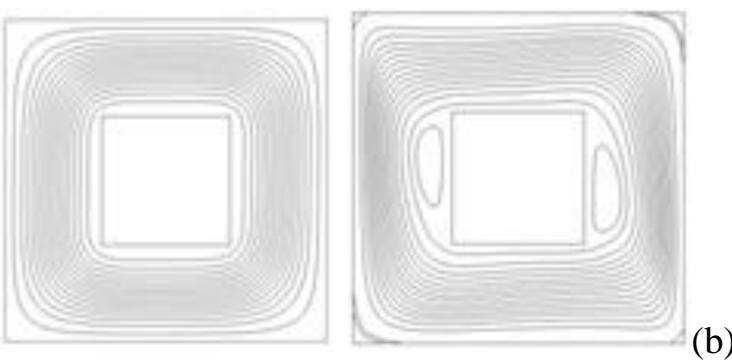

Fig. 2. Streamlines for $R a=$ (a) $10^{3}$ (b) $10^{4}$. (a)

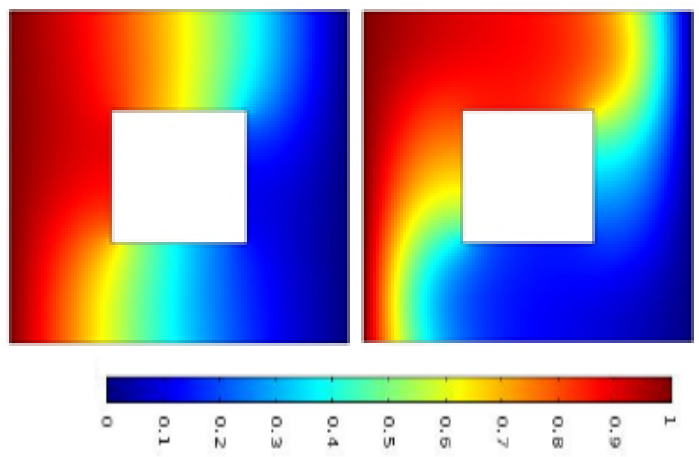

(b)

Fig. 3. Temperature distribution for $R a=$ (a) $10^{3}$ (b) $10^{4}$ (c) Color bar of the temperature distribution.

To further verify the validity of our numerical setup, we considered a geometry representative of a square cavity heated on the left wall and cooled at the right wall, while the top and bottom walls are adiabatic. In this case, there was no obstacle within the enclosure. For various $R a$, by comparing the maximum, minimum, and averaged $\mathrm{Nu}$ along the hot wall of the square cavity obtained in this work with similar solutions of $[12,3,13,14,15]$, an excellent agreement was obtained as shown in Table 1.

TABLE I. ESTIMATED NUSSELT NUMBERS IN AN OBSTACLE-FREE CAVITY

\begin{tabular}{|c|c|c|c|c|c|c|c|}
\hline$R a$ & $N u$ & Present work & Mousa[12] & Barakos et al [3] & $\begin{array}{c}\text { De Vahl } \\
\text { Davis[13] }\end{array}$ & $\begin{array}{l}\text { Markatos and } \\
\text { Pericleous[14] }\end{array}$ & $\begin{array}{c}\text { Fusegi et al } \\
{[15]}\end{array}$ \\
\hline \multirow[t]{3}{*}{$10^{3}$} & $\begin{array}{l}N u(\max ), \\
\text { (at y) }\end{array}$ & $1.506(0.095)$ & $1.5063(0.09)$ & $1.581(0.099)$ & $1.501(0.092)$ & $1.596(0.083)$ & $1.420(0.083)$ \\
\hline & $\begin{array}{l}N u(\min ), \\
\quad(\text { at } y)\end{array}$ & $0.6917(0.991)$ & $0.693(1.000)$ & $0.670(0.994)$ & $0.692(1.000)$ & $0.720(0.993)$ & $0.764(1.000)$ \\
\hline & $\mathrm{Nu}(\operatorname{avg})$ & 1.1145 & 1.1178 & 1.114 & 1.118 & 1.108 & 1.105 \\
\hline \multirow[t]{3}{*}{$10^{4}$} & $\begin{array}{l}N u(\max ), \\
\quad(\text { at } y)\end{array}$ & $3.525(0.143)$ & $3.5306(0.145)$ & $3.539(0.143)$ & $3.528(0.143)$ & $3.482(0.143)$ & $3.652(0.623)$ \\
\hline & $\begin{array}{l}N u(\min ), \\
\quad(\text { at } \mathrm{y})\end{array}$ & $0.585(0.994)$ & $0.588(0.998)$ & $0.583(0.994)$ & $0.586(1.000)$ & $0.643(0.993)$ & $0.611(1.000)$ \\
\hline & $N u$ (avg) & 2.2473 & 2.2447 & 2.245 & 2.243 & 2.201 & 2.302 \\
\hline \multirow[t]{3}{*}{$10^{5}$} & $\begin{array}{l}N u(\max ), \\
\quad(\text { at } y)\end{array}$ & $7.652(0.088)$ & $7.7163(0.085)$ & $7.636(0.085)$ & $7.717(0.081)$ & $7.626(0.083)$ & $7.795(0.083)$ \\
\hline & $\begin{array}{l}N u(\min ), \\
\quad(\text { at } \mathrm{y})\end{array}$ & $0.774(0.998)$ & $0.728(0.998)$ & $0.773(0.999)$ & $0.729(1.000)$ & $0.824(0.993)$ & $0.787(1.000)$ \\
\hline & $\mathrm{Nu}$ (avg) & 4.5638 & 4.5209 & 4.51 & 4.519 & 4.43 & 4.646 \\
\hline \multirow[t]{3}{*}{$10^{6}$} & $\begin{array}{l}N u(\max ), \\
\quad(\text { at } y)\end{array}$ & $17.001(0.035)$ & $17.5001(0.04)$ & $17.442(0.0638)$ & $17.925(0.0378)$ & $17.872(0.0375)$ & $17.670(0.0379)$ \\
\hline & $\begin{array}{l}N u(\min ), \\
\quad(\text { at } \mathrm{y})\end{array}$ & $1.0976(0.996)$ & $0.989(0.999)$ & $1.001(0.999)$ & $0.989(1.000)$ & $1.232(0.993)$ & $1.257(1.000)$ \\
\hline & $N u$ (avg) & 8.8168 & 8.8187 & 8.806 & 8.799 & 8.754 & 9.012 \\
\hline
\end{tabular}




\section{RESULTS AND DISCUSSIONS}

The results from our simulation of convective flow and heat transfer in a square cavity with a mid-cavity heat source are presented in Figs. 4 and 5 respectively. In Fig. 4, isotherms superimposed on temperature distribution are shown for different $R a$ values and AR. For fixed AR, the temperature is distributed almost symmetrically around the heat generating obstacle for low $R a\left(<10^{4}\right)$. For these low $R a$ values, heated fluid is present in both top and bottom halves of the cavity.
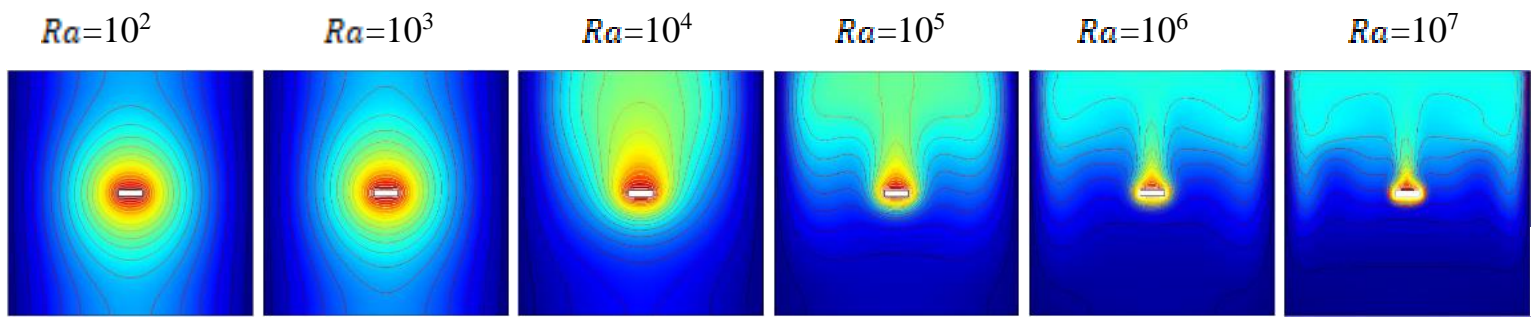

(a)
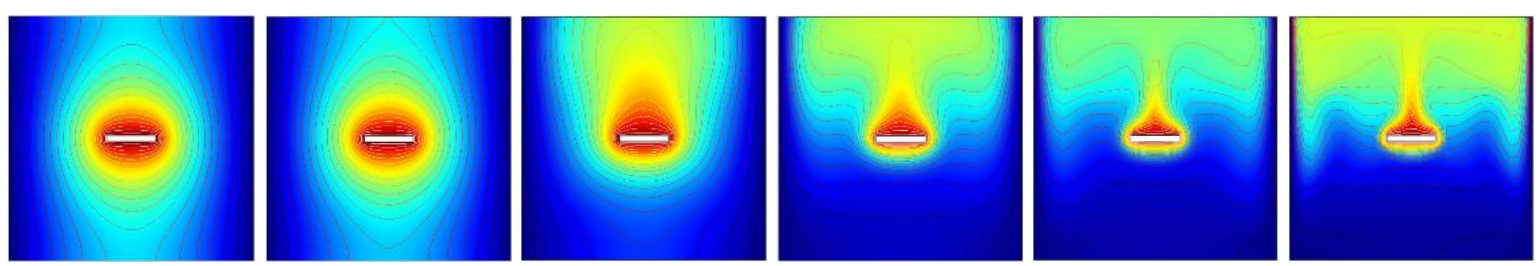

(b)
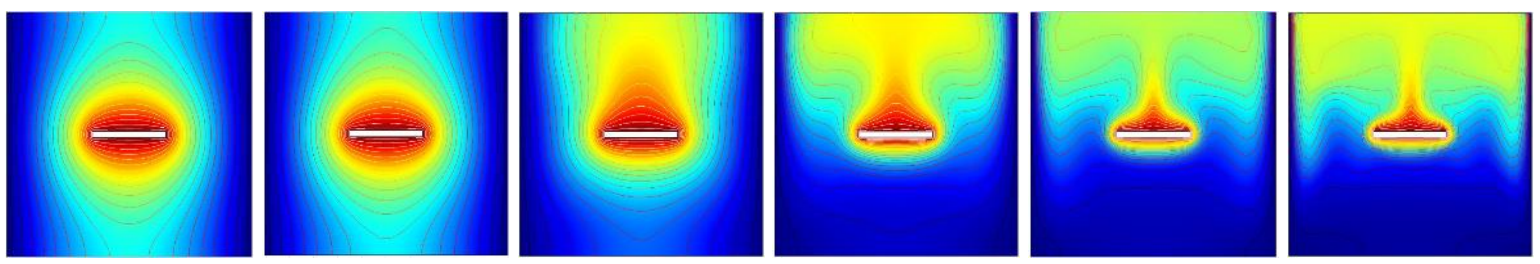

(c)
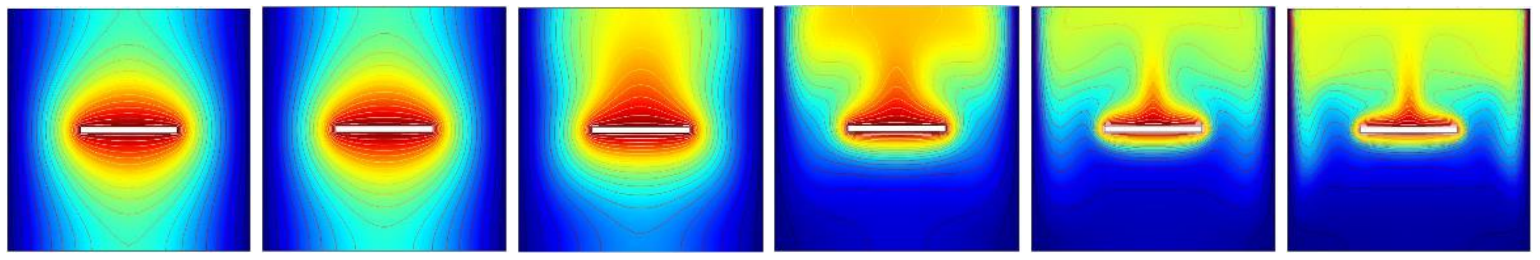

(d)
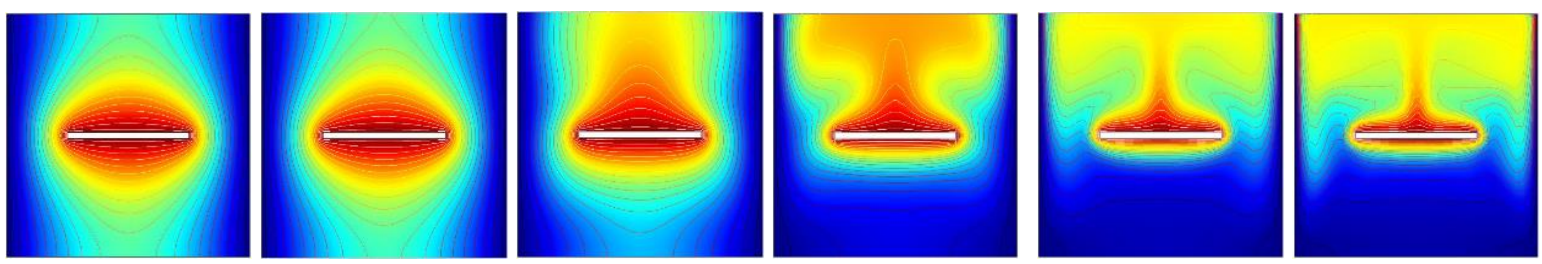

(e)
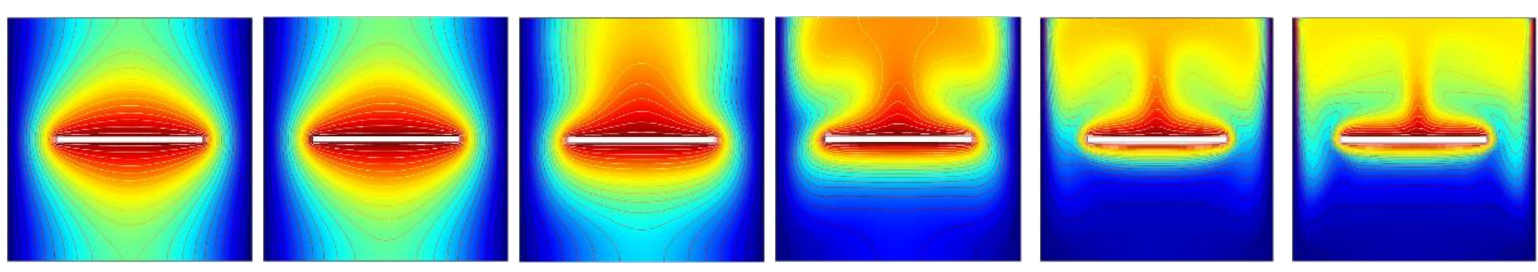

Fig. 4. Variation in Isotherms at various $R a$ for $\mathrm{AR}=$ (a) 0.1, (b) 0.2, (c) 0.3, (d) 0.4, (e) 0.5, (f) 0.6,

(g) Color bar of the temperature distribution. 
Although the fluid around and in contact with the obstacle is heated, for $R a>10^{4}$, due to increasing buoyancy force as the fluid density decreases with temperature, the heated fluid is transported above the obstacle to occupy top insulated wall of the cavity. Therefore, convective heat transfer from the heated obstacle to the fluid is a dominant feature in the top-half of the cavity, while the fluid below the obstacle is generally cold.
As also seen in Fig. 4, with increasing $R a$, the heated fluid ascends along the mid-section of the cavity to spread along the top insulated wall of the cavity. Fluid at the boundary of the vertical cold walls of the cavity are generally cold, however, the flow adjacent to this cold wall can transport cold fluid to the center and top of the cavity.
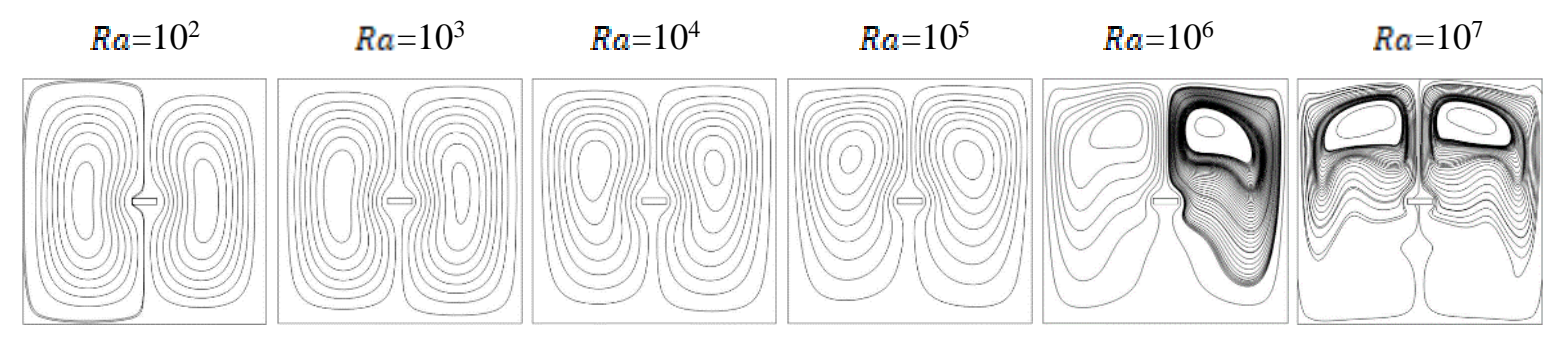

(a)
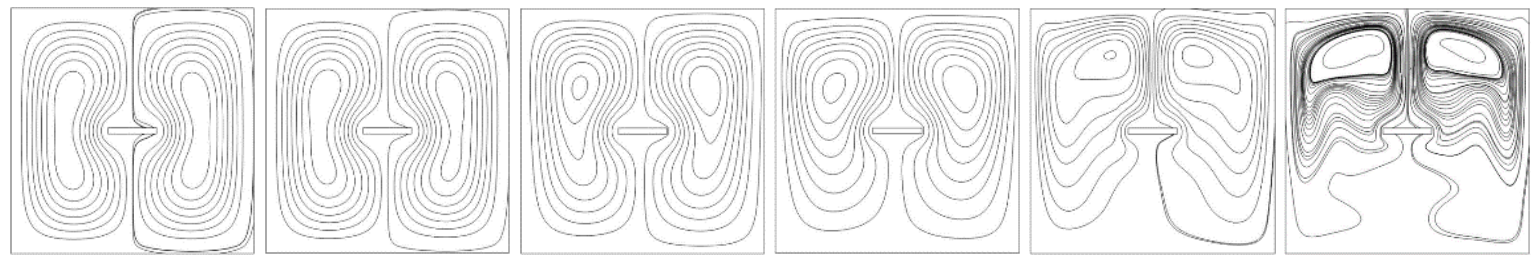

(b)
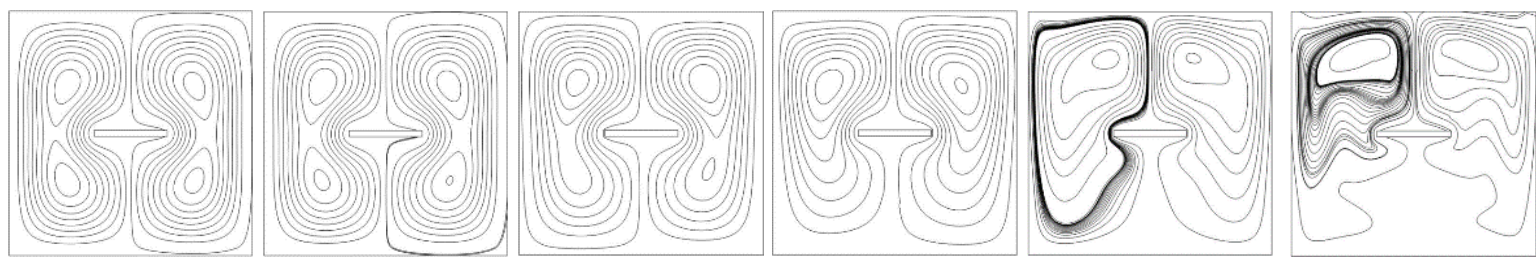

(c)
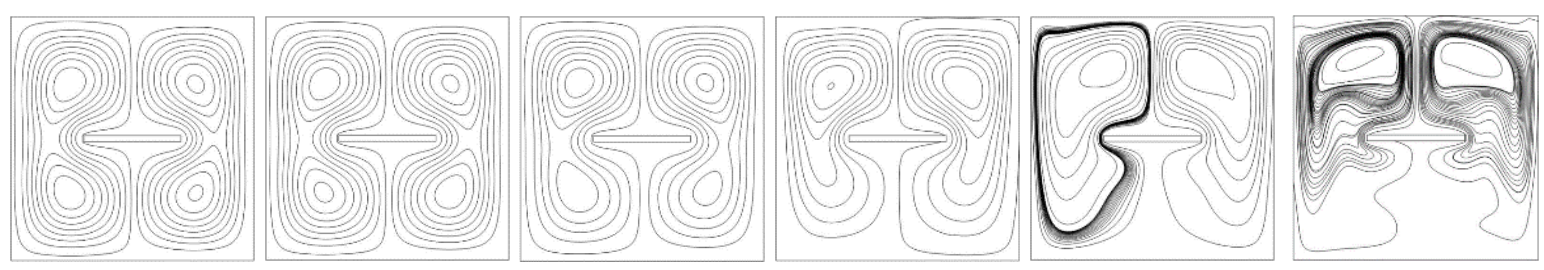

(d)
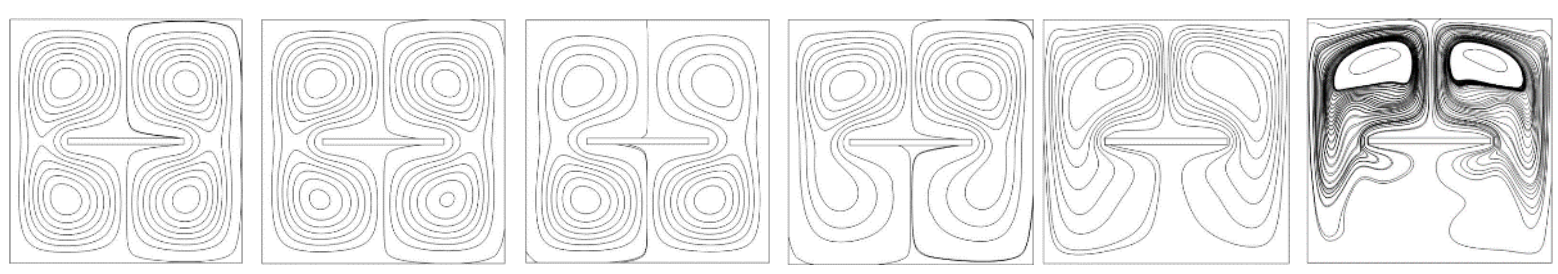

(e)
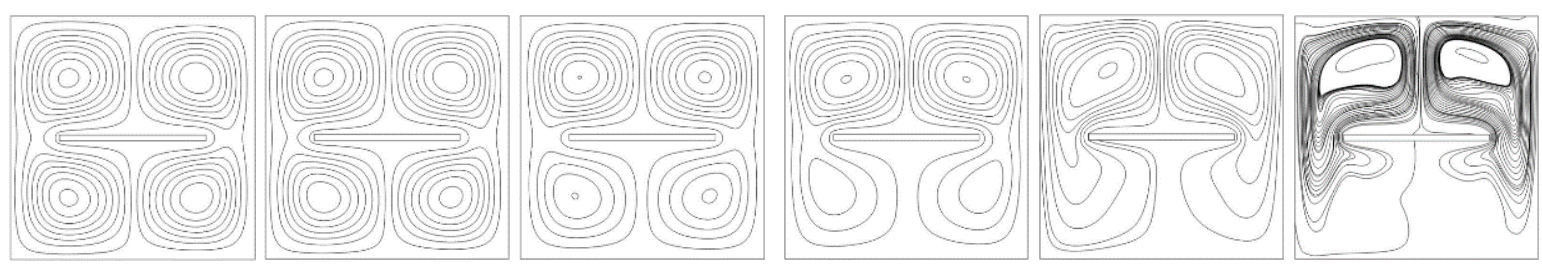

(f)

Fig. 5. Variation of streamlines for various $R a$ for $A R=$ (a) 0.1, (b) 0.2, (c) 0.3, (d) 0.4, (e) 0.5,

(f) 0.6 
For fixed $R a$, this convection of heat in the cavity is enhanced with increasing AR due to the increasing contact area between the heated obstacle and surrounding fluid. Therefore a large volume of fluid expands and becomes less dense due to increasing temperatures which in this case is a function of the area of the heated obstacle. The heat transfer effect is not significant in the lower half of the cavity despite the increase in the AR. In general, similar heat transfer effects in the fluid are observed for a fixed AR with varying $R a$ and vice versa, with this effect being more pronounced at high $R a\left(10^{7}\right)$ and high AR (0.6).

For streamlines in Fig. 5, a double vertical vortex is formed at either side of the obstacle for $\mathrm{AR}=0.1$ to 0.2 and for $R a=$ $10^{2}$ to $10^{5}$. These double vortex further breaks into four vortices with increasing AR. For $R a=10^{6}$ to $10^{7}$, the top half of the cavity is dominated by two vortices which increasingly develops with increasing AR. These dominant vortices can be attributed to increased transport of heated fluid (which is less dense) to the top half of the cavity. As earlier mentioned, the flow adjacent to the cold wall can transport cold fluid to the center and top of the cavity where they are heated.
The absolute velocity $\left(U_{\sigma}\right)$ distributions of the fluid in the cavity at positions $U_{\sigma}(\mathrm{x}, 0.25)$ and $U_{\sigma}(\mathrm{x}, 0.75)$ are plotted in Fig. 6 for different $R a$ with $\mathrm{AR}=0.1$ and 0.6. In the Figure, the absolute velocity has been normalized by the highest recorded value velocity in the cavity that occurs for the case $\mathrm{Ra}=10^{7}$ and $\mathrm{AR}=0.6$. As shown, it can be quickly seen that the flow velocity of fluid at the top half of the cavity is higher than that of the bottom. This is not surprising as the top half is a region characterized with higher temperatures, so with increasing temperatures, the kinetic energy of the fluid molecules rises making these molecules move faster. For any $\mathrm{AR}$, with increasing $R a$, the inconsistent velocity profiles at each $R a$ observed at the bottom of the cavity (Figs. 6(a) and 6(c)) are traceable to the gradual breakdown of the vortices that were significant features at the bottom of the cavity. Conversely, for any AR, the velocity profiles in the top half of the cavity (Figs. 6(b) and 6(d)) are connected to the development of the two significant vortices in the top of the cavity with increasing $R a$.
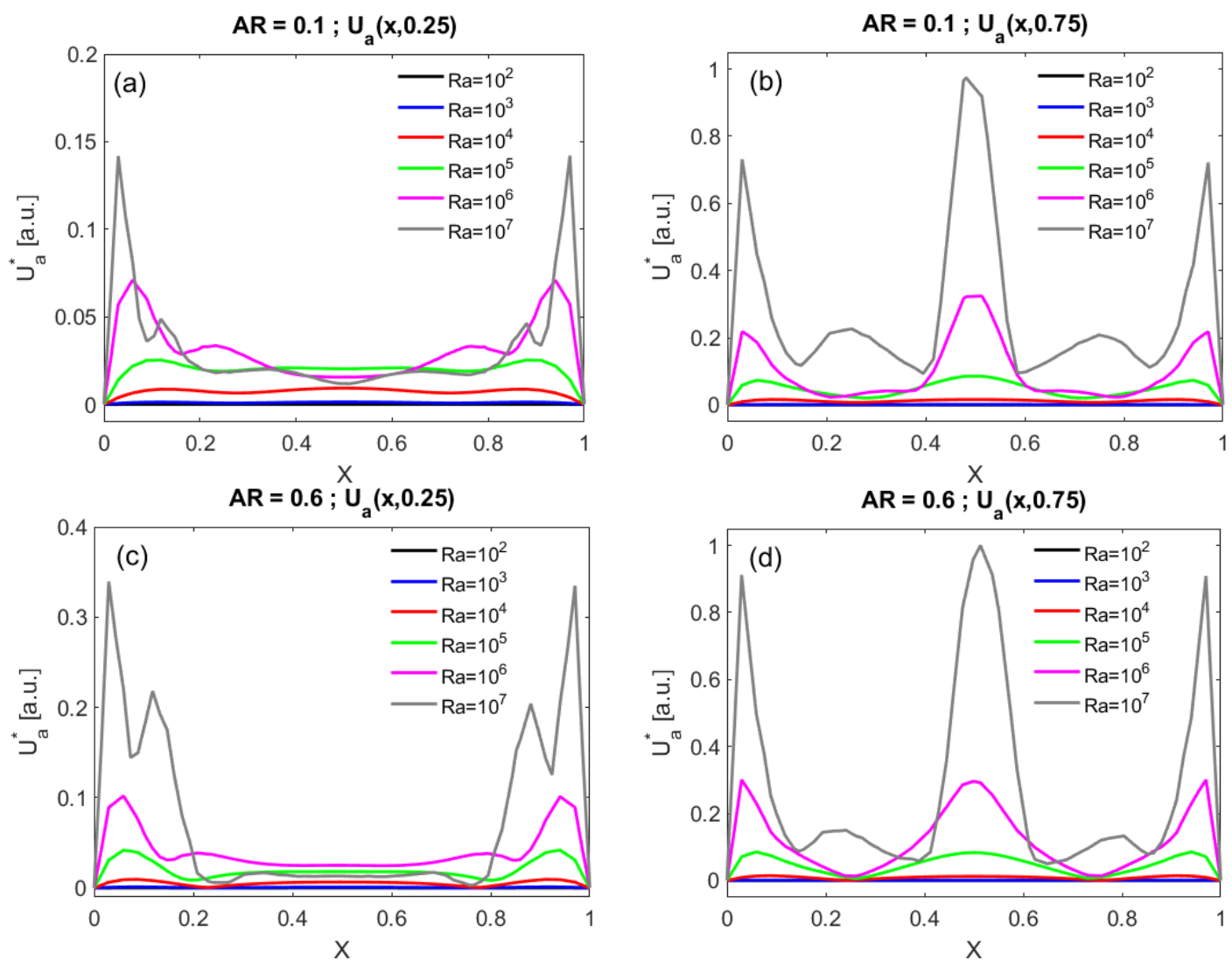

Fig. 6. Variation of $U_{\alpha}$ in the cavity for (a) $\mathrm{AR}=0.1$ at $(x, 0.25)$, (b) $\mathrm{AR}=0.1$ at $(y, 0.75)$

(c) $\mathrm{AR}=0.6$ at $(x, 0.25)$, (d) $\mathrm{AR}=0.1$ at $(y, 0.75)$ for different $R a$. 
Fig. 7 shows the distribution of $\mathrm{Nu}$ in the top-half of the cavity and along the top boundary of the heat generating obstacle of various AR for various $R a$. A dominating feature in the $N u$ distribution for all AR considered is the maximum local $\mathrm{Nu}$ observed at both ends of the obstacle; with the minimum local $N u$ values being observed at the center of the obstacle. This is indicative of the increased heat transfer by convection at the boundary of the obstacle around its edges as cold fluid is readily transported over these edges causing higher temperature gradients than at the center of
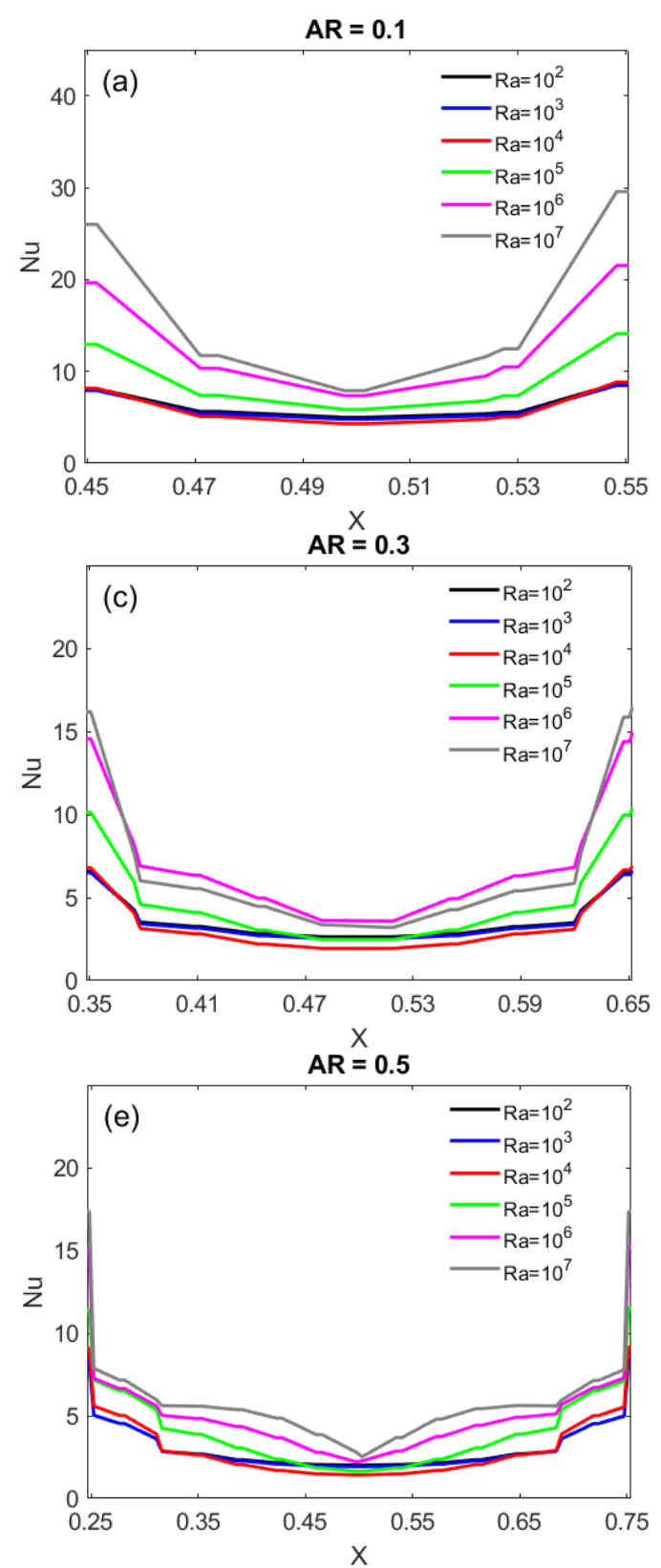

the obstacle. However, towards the center of the obstacle, temperature gradients are lower, indicating decreasing convective heat transfer rates at the surface but an increasing contribution of the heat transfer by conduction from the heated obstacle to the fluid. With increasing AR, the $N u$ distribution stretches along the length of the obstacle as conduction within the fluid in the boundary of the heated obstacle increasingly becomes the dominant mode of transfer of heat. Therefore, the $N u$ generally decreases with increasing AR for all $R a$.
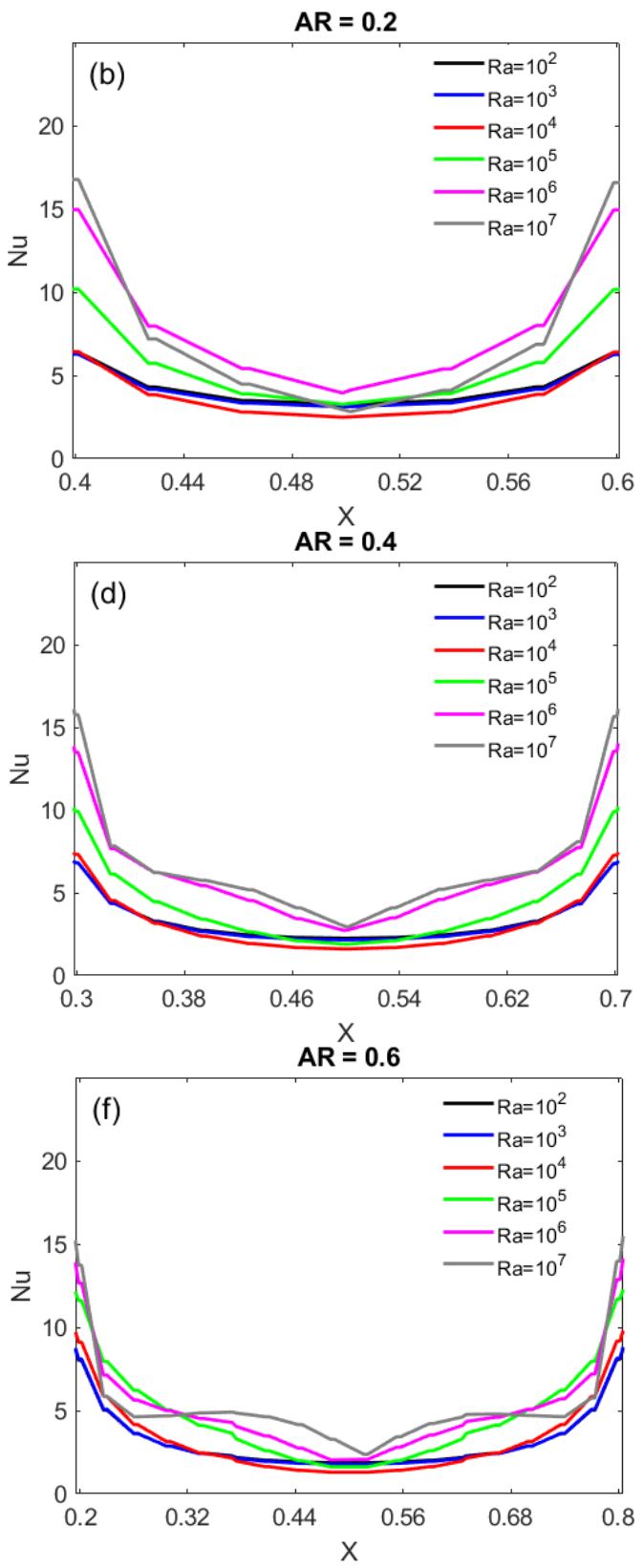

Fig. 7. Variation of $\mathrm{Nu}$ along the boundary of the obstacle with $\mathrm{AR}=$ (a) 0.1 , (b) 0.2 , (c) 0.3 , (d) 0.4 , (e) 0.5 ,

(f) 0.6 for different $R a$. 


\section{CONCLUSIONS}

A numerical study of natural convection in a square cavity with a heat source at its center was carried out using the discontinuous Galerkin weighted residual FDM that the commercial software; COMSOL Multiphysics offers. First, our numerical setup built with this commercial software was validated by considering case studies of matching geometry and boundary conditions available in the literature and the results obtained agree satisfactorily with those found in the literature. Then this study considered a square cavity with a heat generating body of different AR at its center; for this geometry, numerical simulations were conducted with $R a$ in the range, $10^{2}$ to $10^{7}$, and with a heat source of different AR in the range, 0.1 to 0.6 . The results showed that with increasing $R a$, the heated fluid is transported to the top-half of the cavity due to buoyancy forces while the bottom half of the cavity is much cooler. The top-half of the cavity is characterized by increased fluid motion due to convection of the less dense heated fluid. From the estimated $\mathrm{Nu}$ along the boundary of the obstacle in the top-half of the cavity, increased rate of heat transfer by convection is dominant in the vicinity of the edges of the obstacle. However, this decreases towards the center of the obstacle owing to the growing influence of heat transfer by conduction. With increasing $\mathrm{AR}$, heat conduction to the fluid becomes enhanced as a wider area of the obstacle is in contact with the fluid.

\section{NOMENCLATURE}

$\begin{array}{ll}u & \text { Velocity in x-direction } \\ v & \text { Velocity in y-direction } \\ U_{\alpha} & \text { Dimensionless absolute velocity } \\ \Omega & \text { Vorticity } \\ R a & \text { Rayleigh number } \\ P r & \text { Prandtl number } \\ N u & \text { Nusselt number } \\ \propto & \text { Thermal diffusivity } \\ \vartheta & \text { Dynamic viscosity } \\ g & \text { Acceleration due to gravity } \\ \text { Tc } & \text { Cold temperature } \\ \beta & \text { Volumetric thermal expansion } \\ \text { Th } & \text { Hot temperature } \\ P & \text { Pressure } \\ \rho & \text { Density } \\ l & \text { Length of the enclosure } \\ \delta & \text { Width of the enclosure } \\ L & \text { Height of the enclosure } \\ N u(\max ) & \text { Maximum Nusselt number } \\ N u(\min ) & \text { Minimum Nusselt number } \\ N u(a v g) & \text { Average Nusselt number }\end{array}$

\section{REFERENCES}

[1]. Jahirul M. H.M, Bhuiyan A.H., Alim M.A., A numerical study of natural convection in a square enclosure with non-uniformly heated bottom wall and square shape heated block, American Journal of Engineering Research, 2015;4(5):124-132.

[2]. Kitamura K., Mitsuishi A., Suzuki T., Kimura F. Fluid flow and heat transfer of natural convection induced around a vertical row of heated horizontal cylinders, International Journal of Heat and Mass Transfer, 2016; 92: 414-429

[3]. Barakos G and Mitsoulis E, Natural Convection flow in a square cavity revisited: Laminar and Turbulent models with wall functions, International Journal of Numerical Methods in Fluids, 1994; 18: 695-719.

[4]. Abdelmassih G, Vernet A and Pallares J., Numerical simulation of incompressible laminar flow in a three-dimensional channel with a cubical open cavity with a bottom wall heated. Journal of Physics A: Conference Series, 2012;395: 012099.

[5]. Sheikhzadeh G. A., Nikfar M., Fattahi A., Numerical study of natural convection and entropy generation of $\mathrm{Cu}$-water nanofluid around an obstacle in a cavity, Journal of Mechanical Science and Technology 2012; 26 (10): 3347-3356.

[6]. Fusegi, T., Hyun, J. M., Kuwahara, K. Natural convection in a differentially Heated square cavity with internal heat generation, Numerical Heat Transfer, Part A: Applications, 1992; 21: 215-229.

[7]. Lo D C., Young,D . L, Tsai C.C. High resolution of 2D natural convection in cavity by DQ method, Journal of computational and Applied Mathematics, 2004; 203:219-236.

[8]. Basak T., Roy S. and Balakrishnan A.R., Effects of thermal boundary conditions on natural convection flows within square cavity, International Journal of Heat and Mass Transfer, 2006; 49:4525-4535.

[9]. Jong Yun Oh, Man Yeong Ha, Kyung Chun Kim. Numerical study of heat transfer and flow of natural convection in an enclosure with a heat-generating conducting body, Numerical Heat Transfer, Part A: Applications, 2007; 31(3): Pp 289-303.

[10]. Azwadi, C.S.N. and Idris, M.S. Finite different and lattice boltzmann modeling for simulation of natural convection in a square cavity, International Journal of Mechanical and Materials Engineering, 2010; 5 (1): 80-86.

[11]. Bhuiyan H., Islam R., Alim M. A. Magnetohydrodynamic free convection in a square cavity with semicircular heated block, International Journal of Engineering Research \& Technology, 2014:3(11); 675-681.

[12]. Mousa M. M. Modeling of Laminar Buoyancy Convection in a Square Cavity containing an Obstacle, Bulletin of the Malaysian Mathematical Sciences Society, 2013: 38(2);483-498.

[13]. De Vahl Davis G. and Jones I.P. Natural convection of air in a cavity of air in a square cavity, a benchmark numerical solution. International Journal Numerical Methods in Fluids, 1983:3; 249264.

[14]. Markatos N.C. and Pericleous K.A. Laminar and turbulent natural convection in an enclosed cavity, International Journal of Heat and Mass Transfer, 1984:27;775-772.

[15]. Fusegi T., Hyun J.M., Kuwahara K. and Farouk B. A numerical study of three-dimensional natural convection in a differentially heated cubical enclosure, International Journal Numerical Methods in Fluids, 1991:34; 1543-1557. 